\title{
Effect of accumbens nucleus shell lesioning on bitemporal lobe epilepsy in rat model
}

Junwu Fu, Yawei Liu, Kaijun Yang, Hao Long, Kewan Wang, Songtao Qi

Department of Neurosurgery, Nanfang Hospital, Southern Medical University, Guangzhou, Guangdong, China

\begin{abstract}
Introduction: To explore the effect of accumbens nucleus shell (ACbSh) lesioning on bitemporal lobe epilepsy. Material and methods: Adult Wistar rats (male) were enrolled and randomly assigned into the control group and epilepsy groups with multiple time-points. Lithium-pilocarpine was used to establish the rat epilepsy model, while the control group received an equal amount of saline. Ibotenic acid stereotaxic injection was performed to cause accumbens nucleus shell lesioning for specific groups. Cascade software was used for electroencephalogram (EEG) examination. Fluoro-Jade $C$ staining was performed to examine neuronal degeneration.

Results: Latency period of the epilepsy in epilepsy groups was $15.3 \pm 1.1 \mathrm{~min}$, and epilepsy intensity was $4.8 \pm 0.5$ events/ 12 h. ACbSh lesioning significantly reduced aggressive behavior. Compared with epilepsy groups without ACbSh lesioning, ACbSh lesioning significantly decreased epileptic seizures and reduced epileptic duration $(p<0.05)$. EEG showed that there were still sharp waves in the hippocampus and amygdala region after ACbSh lesioning, but epileptic discharge in prefrontal cortex was significantly decreased ( $p<0.05)$, while epilepsy groups without ACbSh lesioning had more sharp waves in the prefrontal cortex, hippocampus and amygdala region. Fluoro-Jade $C$ staining showed that ACbSh lesioning significantly decreased grades of neuronal degeneration $(p<0.05)$.

Conclusions: Recurrent epilepsy caused neuronal degeneration via ACbSh region-related pathways, and ACbSh lesioning could mitigate epilepsy-caused neuronal degeneration by reducing epileptic discharge.
\end{abstract}

Key words: epilepsy, accumbens nucleus shell, electrophysiology, neuronal degeneration.

\section{Introduction}

Epilepsy is one of the most common chronic neurological diseases in the clinical scenario, and repeated outbreaks of epilepsy could cause progressive brain injury, which induces cognitive dysfunction and mental disorders. Clinical trials showed that epilepsy has not only lowered the quality of life, but also caused a great burden on individual life and social economy $[6,17]$. Latest studies indicated that the "abnormal neural network" theory had promising clinical implications on epilepsy treatment [7,21]. Under the conduct of error signals, remnant neurons or new-born neurons in damaged brain tissue generate non-physiological nervous processes, and such abnormal processes established a novel neural network in pathological conditions, which acted as origins or pathologic pathways of epileptiform discharges $[2,19]$.

\section{Communicating authors}

Kewan Wang \& Songtao Qi, Department of Neurosurgery, Nanfang Hospital, Southern Medical University, No. 1838 north

of Guangzhou Avenue, Guangzhou, Guangdong, China, e-mails: kewanwang@126.com; qisongtaosjwk@163.com 
Preclinical studies demonstrated that accumbens nucleus shell (ACbSh) had been involved in the progress of epilepsy. Pilocarpine-induced rat epilepsy models showed that hippocampus had severe degeneration of neurons, while multiple nucleus in thalamus and ACbSh had also suffered from neuronal degeneration [3,23]. However, details were not clear as to the association between ACbSh and epilepsy. In addition, ACbSh lesioning has potential efficacy in the addictive circle, suggesting ACbSh could be a target for abnormal network.

This study was aimed to explore the association between epileptiform discharges and ACbSh lesioning with establishment of lithium-pilocarpine induced bitemporal lobe epilepsy, and further to reveal potential of $\mathrm{ACbSh}$ lesioning in epilepsy treatment.

\section{Material and methods \\ General material}

Adult Wistar rats (male) were purchased from the animal experiment center of the Southern Medical University, weighing $220 \pm 20$ g. Rats were bred in SPF animal laboratory (room temperature $20-24^{\circ} \mathrm{C}$, Xnormal circadian rhythms). High precision stereotaxic instruments were purchased from RWD, China. Electroencephalograph Cascade software/system was produced by Cadwell, USA. Chloral hydrate was purchased from Qinghai province pharmaceutical factory, China. Lithium was purchased from Sigma, USA. Pilocarpine was also purchased from Sigma, USA. All animal treatment and experiments got the ethical approval from the local ethics committee (Nanfang Hospital, Southern Medical University), and were performed according to formal institutional standards.

\section{Fluoro-Jade C staining}

\section{Reperfusing and grouping}

Rats were randomly assigned into the control group and epilepsy groups with multiple time-points. There are five groups of 10 rats each:

- A group, control group with treatment of normal saline;

- B group, lithium-pilocarpine induced bitemporal lobe epilepsy rat models for 3 hours and sham operation;
- C group, lithium-pilocarpine induced bitemporal lobe epilepsy rat models for 3 days and sham operation;

- D group, lithium-pilocarpine induced bitemporal lobe epilepsy rat models for 3 hours and ACbSh lesioning operation;

- E group, lithium-pilocarpine induced bitemporal lobe epilepsy rat models for 3 days and ACbSh lesioning operation.

Brains were harvested from reperfused Wistar rats, and stored in $4 \%$ paraformaldehyde. All samples were fixed at $4^{\circ} \mathrm{C}$ for 24 hours and transferred successively into $20 \%$ sucrose solution and $30 \%$ sucrose solution.

\section{Staining and measurement}

Fluoro-Jade C staining was performed after paraformaldehyde fixing with a routine procedure [2]. Fluoro-Jade C positive cells were assessed with Olympus BX60 fluorescence microscope (Blue filter, excitation wavelength $450-490 \mathrm{~nm}$ ).

\section{EEG examination}

Cascade software was used for electroencephalogram (EEG) examination with a routine procedure [5]. Epileptic discharge was assessed for every group, and multiple regions of brains in rat models were examined.

\section{Lithium-pilocarpine induced epilepsy models}

\section{Preoperative management}

All rats were normally bred before lithium-pilocarpine treatment, and had EEG examination.

\section{Electrodes implantation}

All rats were anesthetized with chloral hydrate (intraperitoneal injection, $400 \mathrm{mg} / \mathrm{kg}$ ). The rat head was fixed on the stereotactic device. Regional coordinates were localized according to The Rat Brain in Stereotaxic Coordinates - Compact Third Edition $[2,19]$. The operative region was established by the dentist's micro-drill. Electrodes were implanted with dental cement.

\section{Induced epilepsy}

Lithium was intraperitoneally injected to rats $(100 \mathrm{mg} / \mathrm{ml})$, and pilocarpine was intraperitoneally 
injected $(10 \mathrm{mg} / \mathrm{ml})$ after 18-20 h [3]. Epilepsy intensity scoring was performed according to Racine graded standard [23].

\section{Accumbens nucleus shell lesioning operation}

ACbSh was localized according to electrodes implantation data. We used a microelectrode and an electrophysiological recording system (Polyview Software from Grass, America).

Electrode coordinates: The rats were placed in a Cosman-Roberts-Wells stereotactic frame, with the base bar parallel to the anterior commissure-posterior commissure line. Bregma and posterior planes were exposed after craniofacial anatomic procedure. The coordinates were located according to the stereotactic map of the rat brain as follows:

- The right prefrontal cortex: $2.5 \mathrm{~mm}$ front to Bregma, $3.5 \mathrm{~mm}$ side to middle line. $3 \mathrm{~mm}$ below dura mater.

- The right nucleus accumbens: $1.6 \mathrm{~mm}$ front to Bregma, $1.0 \mathrm{~mm}$ side to middle line. $7.5 \mathrm{~mm}$ below dura mater.

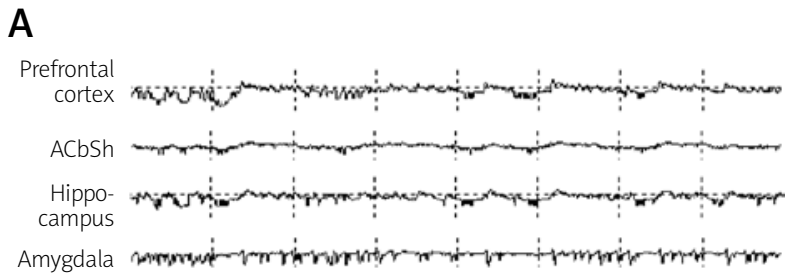

B

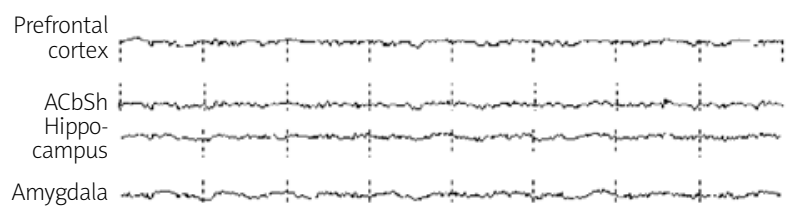

C

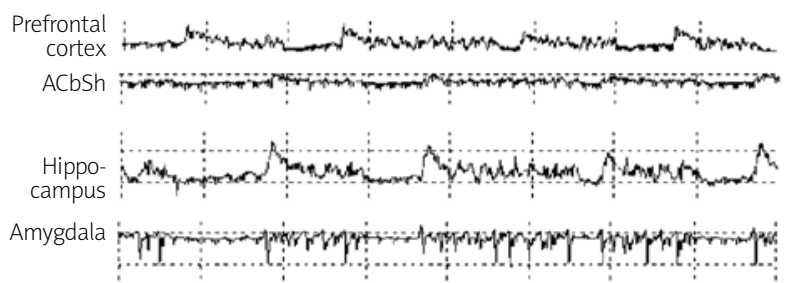

Fig. 1. Electroencephalogram (EEG) data to verify lithium-pilocarpine induced epilepsy. A) EEG data in the first time course. B) EEG data in the first second course. C) EEG data in the control group.
- The right amygdala: $2.8 \mathrm{~mm}$ front to Bregma, $5.0 \mathrm{~mm}$ side to middle line. $8.6 \mathrm{~mm}$ below dura mater.

- The right hippocampus: $4.8 \mathrm{~mm}$ front to Bregma, $5.0 \mathrm{~mm}$ side to middle line. $6.3 \mathrm{~mm}$ below dura mater.

The procedure was performed with a routine dental drill, and electrodes were implanted to four locations (the right prefrontal cortex, the right nucleus accumbens, and the right amygdala) with the guidance of the stereotaxic instrument.

\section{Accumbens nucleus shell lesioning}

$0.2 \mu \mathrm{l}$ ibotenic acid was injected into ACbSh with a micro-injection pump. Ibotenic acid injection should be completed in 15 minutes and the needle should remain in the lesion site for 5 minutes. Electrodes were initiated after ACbSh lesioning, and dental cement was used to seal the operation drill site. EEG was monitored every other day in the first week after operation. Penicillin was administered with intraperitoneal injection at the third day after operation.

\section{Statistical analysis}

SPSS23.0 was used for statistical analysis. All data were expressed with the form of $\pm \mathrm{s}$. All data were performed with $t$ test, $p<0.05$ was considered statistical significance.

\section{Results}

\section{Lithium-pilocarpine induced epilepsy was successfully established}

Epilepsy status was assessed and recorded for all lithium-pilocarpine treated rats. Analysis data showed that the first epilepsy onset latency was $15.3 \pm 1.1 \mathrm{~min}$, and epilepsy intensity was $4.8 \pm 0.5$ events $/ 12 \mathrm{~h}$. The first time course was $13.2 \pm 1.6 \mathrm{~min}$, and the second time course was $77.1 \pm 6.4 \mathrm{~min}$. Total time course was $90.3 \pm 8.0 \mathrm{~min}$. EEG data of multiple regions in brain were collected to verify lithium-pilocarpine induced epilepsy. Compared with normal EEG data in the control group, lithium-pilocarpine treated rats had significantly increased epileptic discharge events (Fig. 1).

\section{Ibotenic acid injection caused ACbSh injury}

HE staining was performed to assess ACbSh injury. Compared with normal ACbSh region, ibotenic 

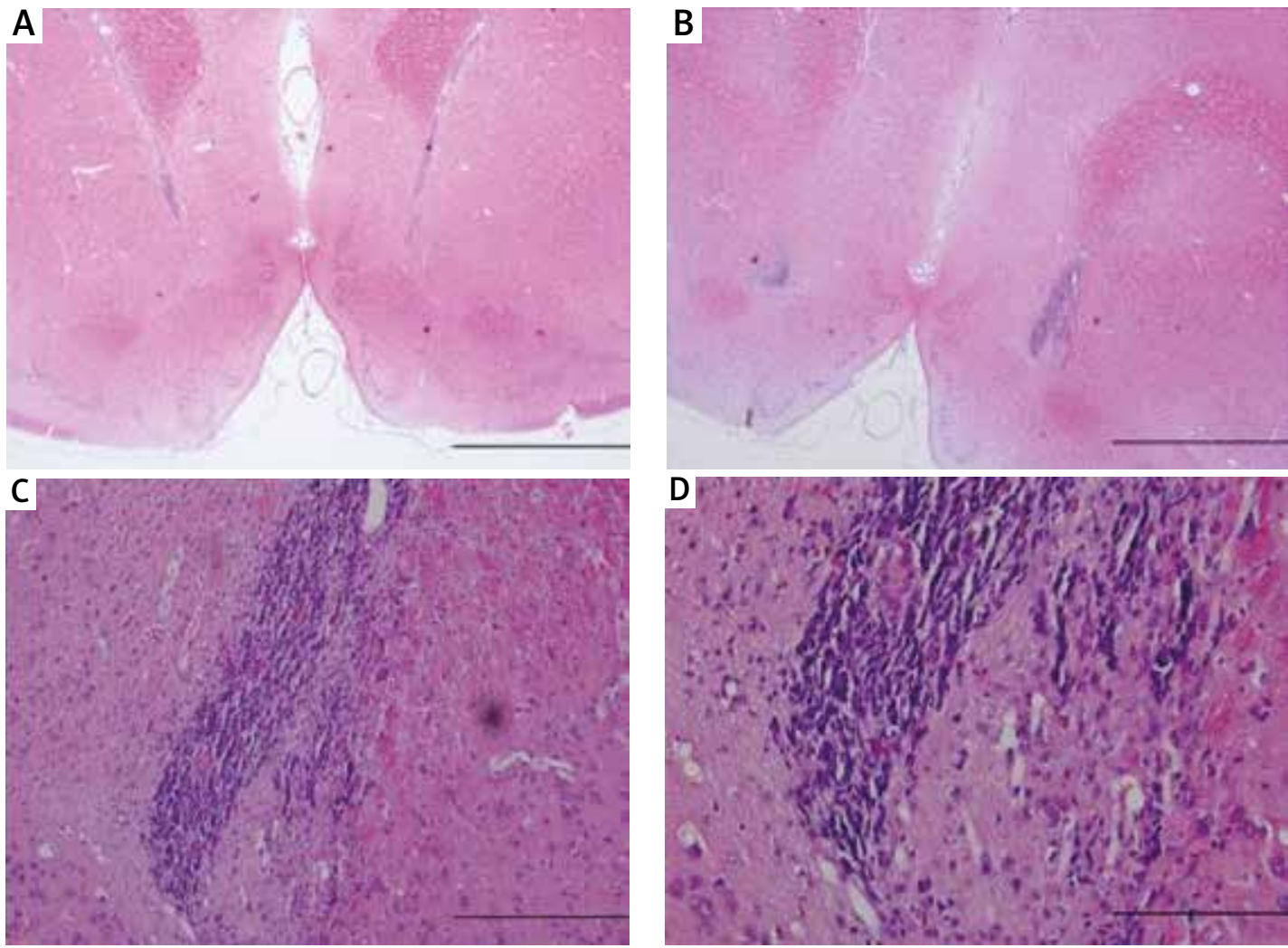

Fig. 2. Hematoxylin and eosin (HE) staining for ibotenic acid injection in accumbens nucleus shell (ACbSh). A) Normal ACbSh region (200x). B) ACbSh region (200x). C) ACbSh region in rats with ibotenic acid injection (200x). D) ACbSh region in rats with ibotenic acid injection (200x).

acid injection indeed caused ACbSh injury, verified by cell lysis, hyaline change, neuronal degeneration, shrunken nucleolus and cellular edema (Fig. 2). This suggested that ACbSh lesioning was successfully performed.

\section{Accumbens nucleus shell lesioning decreased epileptic discharge events}

Further EEG examination was performed to assess efficacy of ACbSh lesioning on epileptic discharge. Compared with lithium-pilocarpine treated rats without $\mathrm{ACbSh}$ lesioning, ACbSh lesioning sig- nificantly alleviated epileptic discharge symptoms, verified in the prefrontal cortex, hippocampus and amygdala regions (Fig. 3).

\section{Accumbens nucleus shell lesioning improved epileptic behavior}

Compared with lithium-pilocarpine treated rats without ACbSh lesioning, ACbSh lesioning significantly reduced epilepsy-induced aggressive behavior events and epilepsy onsets. In addition, ACbSh lesioning shortened epilepsy duration (Table I, $p<0.05)$.

Table I. Epileptic behaviour changes analysis for ACbSh lesioning

\begin{tabular}{lcc}
\hline Grouping & Onsets/12 h & Epilepsy duration (seconds)/onsets \\
\hline ACbSh lesioning groups & $1.50 \pm 1.38$ & $36.50 \pm 11.48$ \\
\hline Sham operation groups & $4.33 \pm 1.03$ & $272.17 \pm 118.35$ \\
\hline$t$ & 4.026 & 4.855 \\
\hline$p$ & 0.0032 & 0.0147 \\
\hline
\end{tabular}


A

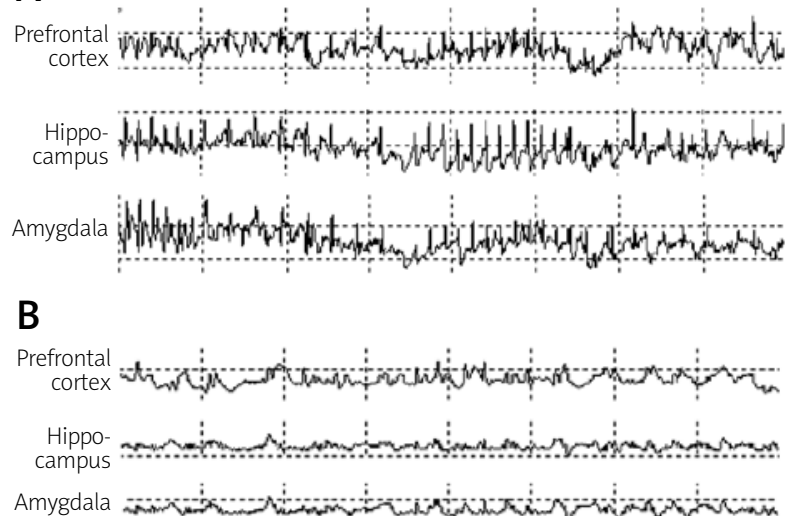

Fig. 3. Electroencephalogram (EEG) data for efficacy of accumbens nucleus shell (ACbSh) lesioning on epileptic discharge events. A) EEG data in lithium-pilocarpine treated rats without $\mathrm{ACbSh}$ lesioning. B) EEG data in lithium-pilocarpine treated rats with $\mathrm{ACbSh}$ lesioning.
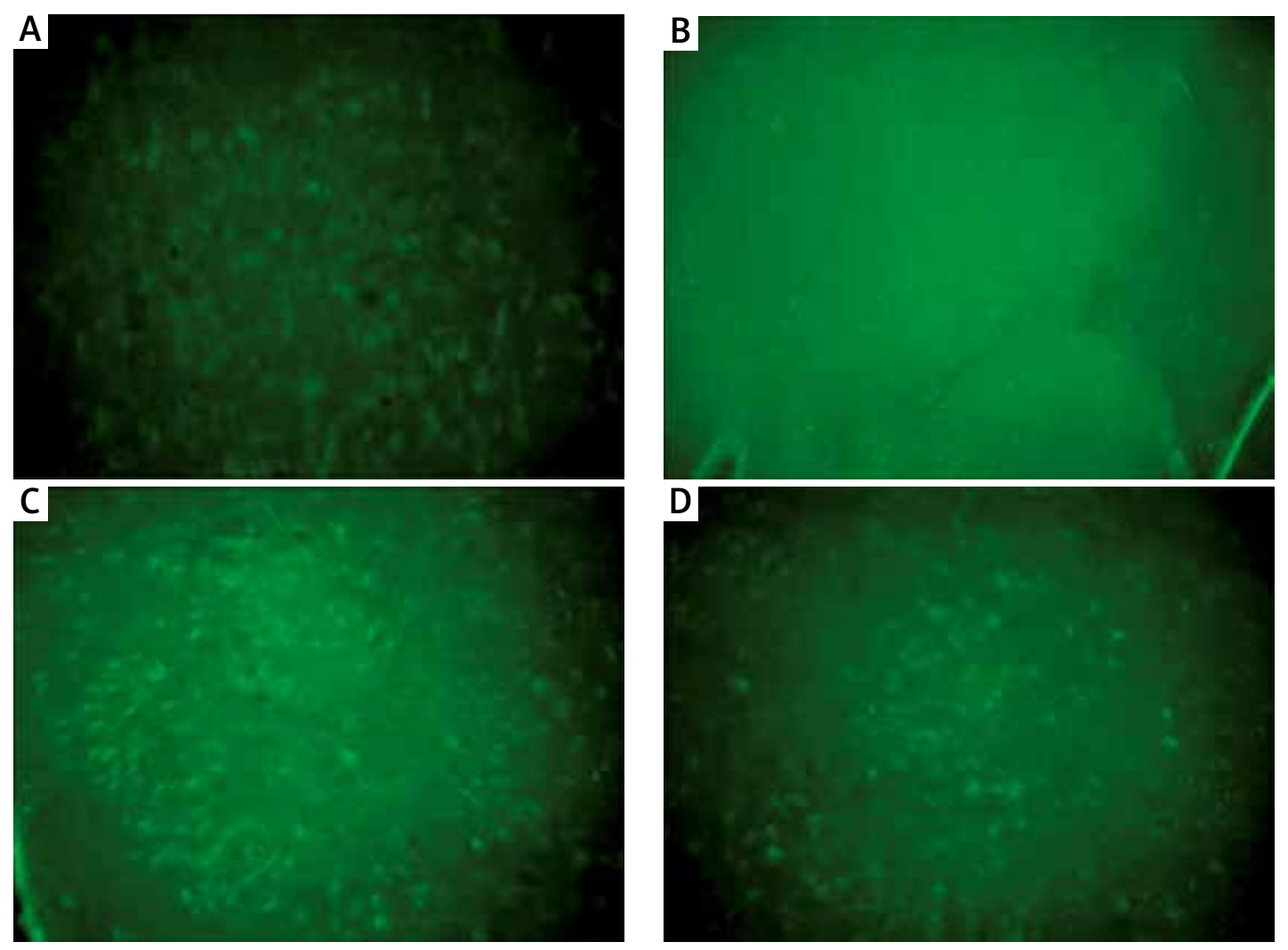

Fig. 4. Fluoro-Jade C staining for groups with different epilepsy duration. A) Denatured neurons staining in rats without accumbens nucleus shell (ACbSh) lesioning (3 hours' epilepsy duration). B) Denatured neurons staining in rats with $\mathrm{ACbSh}$ lesioning (3 hours' epilepsy duration). C) Denatured neurons staining in rats without ACbSh lesioning (3 days' epilepsy duration). D) Denatured neurons staining in rats with ACbSh lesioning (3 days' epilepsy duration) (plotting scale 400x). 

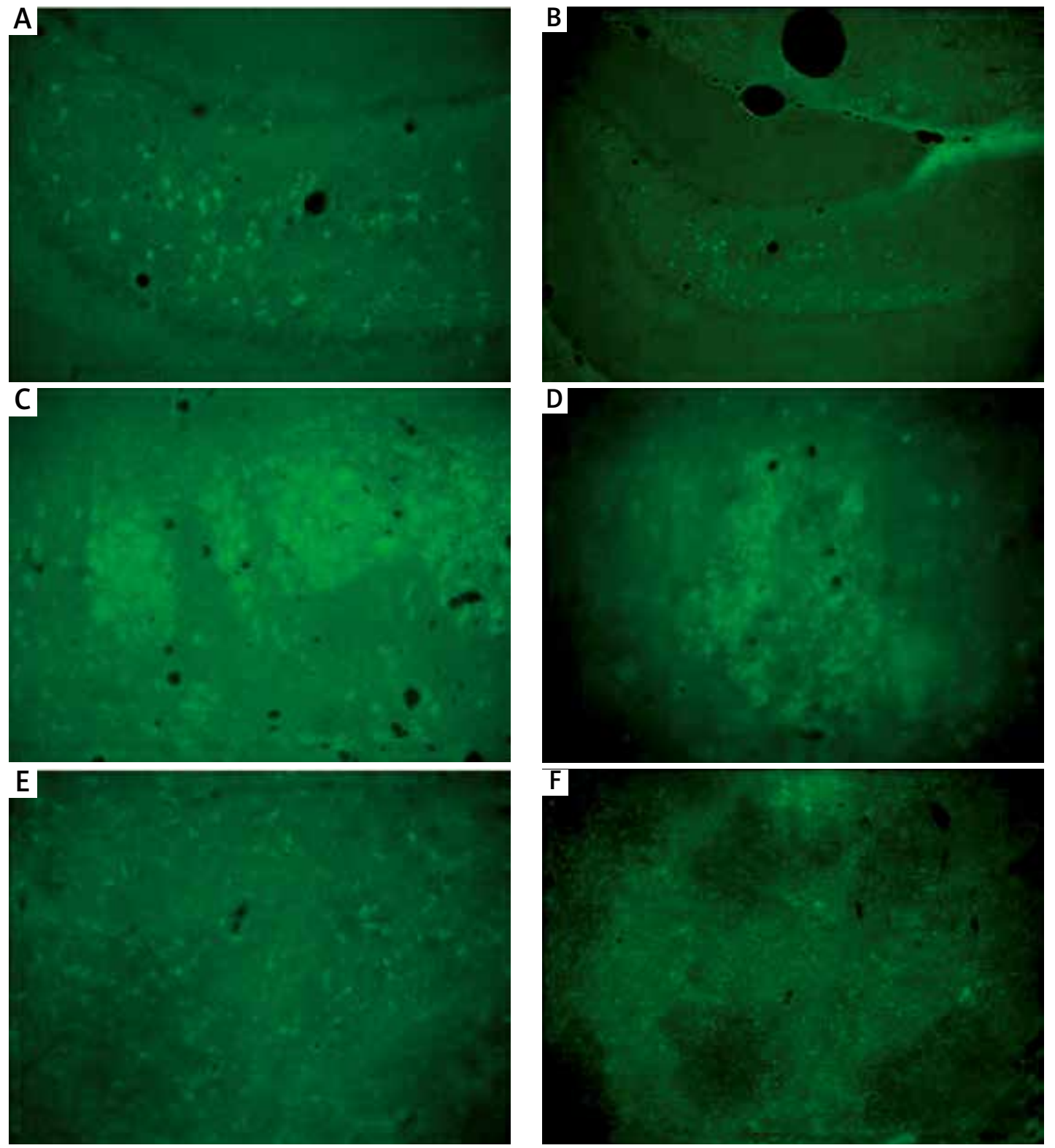

Fig. 5. Fluoro-Jade C staining for groups with different regions. A) Prefrontal cortex neurons staining in rats without accumbens nucleus shell (ACbSh) lesioning. B) Prefrontal cortex neurons staining in rats with ACbSh lesioning. C) Hippocampus neurons staining in rats without ACbSh lesioning. D) Hippocampus neurons staining in rats with ACbSh lesioning. E) Amygdala neurons staining in rats without ACbSh lesioning. F) Amygdala neurons staining in rats with ACbSh lesioning (plotting scale 400x).

Functional neurosurgery was recently considered as a potential therapy for epilepsy-induced disorder, and $\mathrm{ACbSh}$ region was proved with promising efficacy for neurological function. For example, ACbSh region activation was associated with drug dependence $[8,15,23]$. Efferent fibers of ACbSh region were spiny neurons, mainly conducting to multiple regions of midbrain and basal ganglia. Similar to other corpus striatum, ACbSh was also involved in discharge of brain tissue, but it is unclear whether ACbSh influenced development of epilepsy [4,25]. Physiology studies showed that there were many functional domains in ACbSh, which played different roles in human neuron activity $[11,20]$. Inside putamen 
of ACbSh mainly induces cognition of new things, foraging behavior, reward activity and drug dependence. Nucleus of accumbens was possibly associated with spatial learning, conditioned reflex and impulsive choice. Accordingly, ACbSh was regarded as a novel drug target for nerve diseases.

Bitemporal lobe epilepsy was a severe nerve disease which temporally has no optimal treatment, thus our study was aimed to explore whether ACbSh could be associated with bitemporal lobe epilepsy via establishing a rat epilepsy model. The previous study indicated that abnormal expression of c-fos firstly occurred in ACbSh, piriform cortex, prefrontal lobe and corpus striatum in pentetrazol repeated kindling induced epilepsy model $[22,24,26]$. It is also reported that dopamine receptor agonists injection in ACbSh inhibited absence epilepsy onset $[1,13]$. Intracranial electrode clinical trial demonstrated that epileptic discharge in medial temporal lobe epilepsy patients firstly was conducted from medial temporal lobe to medial frontal cortex, while electrical stimulation of ACbSh could reduce seizure frequency $[9,18]$. Our study firstly revealed that ACbSh lesioning was a more efficient treatment for bitemporal lobe epilepsy treatment, verified by shorter epilepsy duration and reduced epileptic discharge events. Moreover, we found that ACbSh lesioning mitigated epilepsy symptoms in multiple regions of the brain, and such effect is independent of epilepsy duration. Intriguingly, previous studies have revealed that some drug might have induced the inhibiting role for ACbSh $[10,16]$, which provided another vision on application prospect of ACbSh lesioning, but these drugs were still under pre-clinical stages [5].

We further explored the detailed mechanisms of ACbSh lesioning in epilepsy. EEG showed that epilepsy onset events and duration was improved after the ibotenic acid injection, but hippocampus and amygdaloid nucleus still had sharp waves, suggesting that ACbSh did not directly take part in epileptic discharge conduction but had some regulatory effect on epileptic events. Fluoro-Jade C staining showed that ACbSh lesioning significantly alleviated neuronal degeneration in multiple regions, including prefrontal cortex, hippocampus and amygdala. This suggested that ACbSh region-related pathways were associated with epilepsy progress, and further experiments were needed to explore details and potential targets in ACbSh.
Although we did not explore details of the protein marker of neuronal degeneration for ACbSh, previous reports suggested that dopaminergic neuronal degeneration played a pivotal role for ACbSh. Moreover, dopamine neuronal loss was a novel concept for targeting treatment for neuronal degeneration, while dopamine is rich in the ACbSh region [12]. Accordingly, we hypothesized that ACbSh lesioning attenuated production of dopamine, which further controlled dopamine-induced neuronal degeneration. Intriguingly, accumbens nucleus shell lesioning was proved with promising efficacy in abstinence addiction therapy [14]. In addition, the dopamine system was involved in the addictive circle, which shared the same mechanism with epilepsy-caused neuronal degeneration. However, our study demonstrated that ACbSh lesioning also reduced epileptic discharge, which was not reported in abstinence addiction therapy.

\section{Conclusions}

Recurrent epilepsy caused neuronal degeneration via ACbSh region-related pathways, and ACbSh lesioning could mitigate epilepsy-caused neuronal degeneration by reducing epileptic discharge.

\section{Disclosure}

The authors report no conflict of interest.

\section{References}

1. Bedwell J, Gooding D, Chan C, Trachik B. Anhedonia in the age of RDoC. Schizophr Res 2014; 160: 226-227.

2. Bonilha L, Helpern J, Sainju R, Nesland T, Edwards J, Glazier S, Tabesh A. Presurgical connectome and postsurgical seizure control in temporal lobe epilepsy. Neurology 2013; 81: 17041710.

3. Chen L, Feng H, Mao X, Ye Q, Zeng L. One hour of pilocarpineinduced status epilepticus is sufficient to develop chronic epilepsy in mice, and is associated with mossy fiber sprouting but not neuronal death. Neurosci Bull 2013; 29: 295-302.

4. Chen N, Yan N, Liu C, Ge Y, Zhang JG, Meng FG. Neuroprotective effects of electrical stimulation of the anterior nucleus of the thalamus for hippocampus neurons in intractable epilepsy. Medical Hypotheses 2013; 80: 517-519.

5. ElBatsh M, Assareh N, Marsden C, Kendall D. Anxiogenic-like effects of chronic cannabidiol administration in rats. Psychopharmacology (Berl) 2012; 221: 239-247.

6. Engel J Jr, McDermott MP, Wiebe S, Langfitt JT, Stern JM, Dewar S, Sperling MR, Gardiner I, Erba G, Fried I, Jacobs M, Vinters HV, Mintzer S, Kieburtz K. Early surgical therapy for drug-resistant 
temporal lobe epilepsy: a randomized trial. JAMA 2012; 307: 922-930.

7. Fisher R, Acevedo C, Arzimanoglou A, Bogacz A, Cross J, Elger C, Engel J Jr, Forsgren L, French J, Glynn M, Hesdorffer D, Lee B, Mathern G, Moshé S, Perucca E, Scheffer IE, Tomson T, Watanabe M, Wiebe S. ILAE official report: a practical clinical definition of epilepsy. Epilepsia 2014; 55: 475-482.

8. Ge Y, Hu W, Liu C, Zhang JG, Meng FG. Brain stimulation for treatment of refractory epilepsy. Chinese Medical Journal 2013; 126: 3364-3370.

9. Goodwin G, Yacko H. Emergence of the exploratory motive in rats. Dev Psychobiol 2004; 45: 34-48.

10. Guimaraes F, Chiaretti T, Graeff F, Zuardi A. Antianxiety effect of cannabidiol in the elevated plus-maze. Psychopharmacology (Berl) 1990; 100: 558-559.

11. Lee K, Shon Y, Cho C. Long-term outcome of anterior thalamic nucleus stimulation for intractable epilepsy. Stereotactic and Functional Neurosurgery 2012; 90: 379-385.

12. Lee Y, Oh JS, Chung SJ, Chung SJ, Kim SJ, Nam CM, Lee PH, Kim JS, Sohn YH. Does smoking impact dopamine neuronal loss in de novo Parkinson disease? Ann Neurol 2017; 82: 850-854.

13. Leweke F, Piomelli D, Pahlisch F, Muhl D, Gerth C, Hoyer C. Symptoms of schizophrenia. Transl Psychiatry 2012; 2: e94.

14. Li N, Wang J, Wang XL, Chang CW, Ge SN, Gao L, Wu HM, Zhao HK, Geng N, Gao GD. Nucleus accumbens surgery for addiction. World Neurosurg 2013; 80: S28.e9-19.

15. Min B, Guoming L, Jian Z. Treatment of mesial temporal lobe epilepsy with amygdalohippocampal stimulation: A case series and review of the literature. Exp Ther Med 2013; 5: 1264-1268.

16. Moreira F, Guimaraes F. Cannabidiol inhibits the hyperlocomotion induced by psychotomimetic drugs in mice. Eur J Pharmacol 2005; 512: 199-205.

17. Moshé SL, Perucca E, Ryvlin P, Tomson T. Epilepsy: new advances. Lancet 2015; 385: 884-898.

18. Pellow S, Chopin P, File SE, Briley M. Validation of open: closed arm entries in an elevated plus-maze as a measure of anxiety in the rat. J Neurosci Methods 1985; 14: 149-167.

19. Richardson M. Large scale brain models of epilepsy: dynamics meets connectomics. J Neurol Neurosurg Psychiatry 2012; 83: 1238-1248.

20. Schmitt F, Voges J, Heinze H, Zaehle T, Holtkamp M, Kowski A. Safety and feasibility of nucleus accumbens stimulation in five patients with epilepsy. J Neurol 2014; 261: 1477-1484.

21. Serletis D, Bulacio J, Bingaman W, Najm I, González-Martínez J. The stereotactic approach for mapping epileptic networks: a prospective study of 200 patients. J Neurosurg 2014; 121: 1239-1246.

22. Shoval G, Shbiro L, Hershkovitz L, Hazut N, Zalsman G, Mechoulam R, Weller A. Prohedonic Effect of Cannabidiol in a Rat Model of Depression. Neuropsychobiology 2016; 73: 123-129.

23. Sprengers M, Vonck K, Carrette E, Marson AG, Boon P. Deep brain and cortical stimulation for epilepsy. Cochrane Database Syst Rev 2014; 6: CD008497.

24. Stam CJ. Modern network science of neurological disorders. Nature reviews. Neuroscience 2014; 15: 683-695.
25. Tykocki T, Mandat T, Kornakiewicz A, Koziara H, Nauman P. Deep brain stimulation for refractory epilepsy. Arch Med Sci 2012; 8: 805-816.

26. Vinod K, Xie S, Psychoyos D, Hungund B, Cooper T, Tejani-Butt S. Dysfunction in fatty acid amide hydrolase is associated with depressive-like behavior in Wistar Kyoto rats. PLoS One 2012; 7: e36743. 